\title{
Citrate targets FBPase and constitutes an emerging novel approach for cancer therapy
}

\author{
Philippe Icard ${ }^{1}$, Ludovic Fournel $2^{2^{*}} \mathbb{0}$, Antoine Coquerel ${ }^{3}$, Joseph Gligorov ${ }^{4}$, Marco Alifano ${ }^{2}$ and Hubert Lincet ${ }^{5}$
}

\begin{abstract}
Gao-Min Liu and Yao-Ming Zhang recently published a review entitled «Targeting FBPase is an emerging novel approach for cancer therapy» (Liu and Zhang in Cancer Cell Int 18:36, 2018). In this paper, the authors highlighted how the down regulation or inactivation of FBPase, a rate limiting enzyme of gluconeogenesis, can promote the Warburg effect and cancer growth. In contrast, activation of this enzyme demonstrates anti-cancer effects and may appear as emerging novel approach for cancer therapy. Among the potential activators of FBP listed by Liu and Zhang, citrate was surprisingly not mentioned although it is an activator of FBPase, also demonstrating various anti-cancer effects in pre-clinical studies. Thus, citrate should be tested as a new therapeutic strategy, in particular in clinical studies.
\end{abstract}

Keywords: Warburg effect, Cancer metabolism, Citrate, FBPase, Cancer therapy

Drs. Gao-Min Liu and Yao-Ming Zhang must be congratulated for their review «Targeting FBPase is an emerging novel approach for cancer therapy» explaining how the down expression or inactivation of this rate limiting enzyme of gluconeogenesis promotes aerobic glycolysis, cancer cell proliferation and dedifferentiation. Therefore, activation of FBPase may have anti-cancer effects, counteracts the Warburg effect and antagonizes chemoresistance [1]. Among the potential activators of FBP listed by Liu and Zhang, citrate was surprisingly not mentioned, although this molecule plays a central role in the metabolism of cancer cells [2]. Physiologically, when energy stores are elevated, citrate is abundant and the activity of (phosphofructokinase 1) PFK1 is nearly switched off [2]. Citrate is also a potent allosteric inhibitor of PFK2 (also called PFKFB (6-phosphofructo-2-kinase/fructose2,6-biphosphatase) in non-cancer cells (such as cardiac cells [3]) and ascites cancer cells [4]. It is likely that citrate also indirectly inhibits PFK2 through an increased palmitate synthesis [5].

*Correspondence: ludovic.fournel@aphp.fr

${ }^{2}$ Thoracic Surgery Department, Cochin Hospital, Paris Descartes University, Unité Inserm 1007, 27 rue du Faubourg Saint Jacques, 75014 Paris, France

Full list of author information is available at the end of the article
By regulating key regulatory enzymes located at entrance and/or exit of glycolysis (PFK1, PFK2, PK), TCA cycle (PDH, SDH), gluconeogenesis (F1,6BPase) and fatty acid synthesis (ACC), citrate controls anabolic and catabolic pathways, and allows a close adjustment of metabolic flows to ATP production [2].

In cancer cells, the Warburg effect is associated with a decrease production of citrate by mitochondria [6]. This reprogramming metabolism is promoted by overexpression of HIF-1alpha and Myc, activation of Ras, loss of functional p53, all factors promoting the down-regulation of mitochondria [6]. Such down-regulation leads to a reduced production of citrate, ATP and $\mathrm{CO}_{2}$, such condition promoting intracellular alkaline $\mathrm{pH}$, a strong activator of PFK-1 [6]. This rate-limiting enzyme of glycolysis is also controlled by a family of regulatory bifunctional enzymes PFKFB. When the kinase activity is promoted, fructose-2,6-bisphosphate (F2,6BP) increases and allosterically activates PFK-1, directing the carbon flux into glycolysis and sustaining cell survival and proliferation [7].

We showed for the first time that administration of citrate to cancer cells arrests cell growth, induces apoptosis (in particular through extinction of expression of the anti-apoptotic factor Mcl-1) and sensitizes chemoresistant cells to cisplatin [8]. Several authors have confirmed 
on different cancer cell lines that citrate administration (generally at $\geq 10 \mathrm{mM}$ ) inhibits glycolysis and ATP production, promotes apoptosis through activation of caspases, and increases response to $\mathrm{Bcl}-\mathrm{xL}$ inhibitors (for additional references see [6,9]). Recently, Ren et al. [9] confirmed that citrate decreases resistance to cisplatin (in particular by reducing the expression of Snail and MUC1 ), and showed that it inhibits the proliferative IGF-1R/ AKT axis, stimulates the suppressive PTEN-eIF2 $\alpha$ pathway, and induces tumor cell differentiation (expression of E-cadherin). Importantly, these authors observed that daily oral administration of citrate at a dose of $8 \mathrm{~g} / \mathrm{kg} / \mathrm{day}$ for 1 month reduces tumor growth of several xenograft tumor models in mice (pancreatic cancer, Ras-driven lung tumor and Her2/Neu mammary cancer), increasing significantly the number of infiltrating T-cells in tumors [9].

All these experimental in vitro and in vivo studies give arguments to consider that high-dose citrate administration inhibits cancer cell development. Nonetheless, the mechanisms underlying this process remain unclear. Beside its allosteric inactivation of PFK 1 and 2, reducing or arresting the glycolytic flux, citrate may also exercise other anti-cancer effects by reducing the levels of fructose-1,6-bisphosphate (F1,6BP) and F2,6BP. Indeed, these molecules couple the glycolytic flux with activation of Ras and cell cycle progression, respectively: (i) cytosolic F1,6BP binds Son of sevenless homolog 1 (Sos1), a factor promoting the activation of Ras and its downstream targets MEK and ERK [10]; (ii) F2,6BP represses p27Kip1, a potent inhibitor of cyclins D and E regulating G1/S transition, and cyclin-dependent kinase (Cdk)-1 regulating entrance in mitosis [7].

Knowing that both isoforms of FBP (FBP1 and FBP2) are inhibited by $\mathrm{Ca}^{2+}$, very probably high concentration of citrate also inactivates these enzymes by this mechanism because citrate is a well-known chelator of calcium. This action should be particularly interesting for treatment of cancers with poor prognosis (such as gastric cancer, brain metastatic breast cancers) expressing low FBP2, because as explained by Liu and Zhang [1], FBP2 is 1000 times more sensitive to inhibition by $\mathrm{Ca}^{2+}$.

More studies are needed to nail down the mechanisms underlying citrate effect in cancer cells proliferation and strengthen the proof of principle.

\footnotetext{
Authors' contributions

Pl: conception, writing of the commentary and revision. LF: conception, editing and submission. AC: conception and commentary design, reviewing. JG: commentary design and correction. MA: conception and reviewing. $\mathrm{HL}$ : design, final supervision. All authors read and approved the final manuscript.
}

\section{Author details}

${ }^{1}$ Thoracic Surgery Department, Cochin Hospital, Normandy University of Caen, Unité Inserm 1199, Paris, France. ${ }^{2}$ Thoracic Surgery Department,
Cochin Hospital, Paris Descartes University, Unité Inserm 1007, 27 rue du Faubourg Saint Jacques, 75014 Paris, France. ${ }^{3}$ Normandy University of Caen, Unité Inserm 1075, Caen, France. ${ }^{4}$ Oncology Department, Tenon Hospital, Pierre et Marie Curie University, Paris, France. ${ }^{5}$ Faculty of Pharmacy of Lyon, CRCL Lyon, Unité Inserm 1052, CNRS 5286, Lyon, France.

\section{Acknowledgements}

Not applicable.

\section{Competing interests}

The authors declare that they have no competing interests.

\section{Availability of data and materials \\ Not applicable.}

\section{Consent for publication}

Not applicable.

\section{Ethics approval and consent to participate}

Not applicable.

\section{Funding}

No funding was provided for this commentary.

\section{Publisher's Note}

Springer Nature remains neutral with regard to jurisdictional claims in published maps and institutional affiliations.

Received: 13 August 2018 Accepted: 4 November 2018 Published online: 08 November 2018

\section{References}

1. Liu GM, Zhang YM. Targeting FBPase is an emerging novel approach for cancer therapy. Cancer Cell Int. 2018;18:36.

2. Icard P, Poulain L, Lincet $H$. Understanding the central role of citrate in the metabolism of cancer cells. Biochim Biophys Acta. 2012;1825:111-6.

3. Crochet RB, Kim JD, Lee H, Yim YS, Kim SG, Neau D, Lee YH. Crystal structure of heart 6-phosphofructo-2-kinase/fructose-2,6-bisphosphatase (PFKFB2) and the inhibitory influence of citrate on substrate binding. Proteins. 2017:85:117-24.

4. Nissler K, Petermann H, Wenz I, Brox D. Fructose 2,6-bisphosphate metabolism in Ehrlich ascites tumor cells. J Cancer Res Clin Oncol. 1995; 121:739-45.

5. Hue L, Maisin L, Rider MH. Palmitate inhibits liver glycolysis. Involvement of fructose 2,6-bisphosphate in the glucose/fatty acid cycle. Biochem J. 1988;251:541-5.

6. Icard $\mathrm{P}$, Lincet $\mathrm{H}$. The reduced concentration of citrate in cancer cells: an indicator of cancer aggressiveness and a possible therapeutic target. Drug Resist Updates. 2016;29:47-53.

7. Yalcin A, Clem BF, Simmons A, Lane A, Nelson K, Clem AL, et al. Nuclear targeting of 6-phosphofructo-2-kinase (PFKFB3) increases proliferation via cyclin-dependent kinases. J Biol Chem. 2009;284:24223-32.

8. Zhang X, Varin E, Allouche S, Lu Y, Poulain L, Icard P. Effect of citrate on malignant pleural mesothelioma cells: a synergistic effect with cisplatin. Anticancer Res. 2009;29:1249-54.

9. Ren JG, Seth P, Ye H, Guo K, Hanai JL, Husain Z, Sukhatme VP. Citrate suppresses tumor growth in multiple models through inhibition of glycolysis, the tricarboxylic acid cycle and the IGF-1R pathway. Sci Rep. 2017;7:4537.

10. Peeters K, Van Leemputte F, Fischer B, Bonini BM, Quezada H, Tsytlonok M, et al. Fructose-1,6-bisphosphate couples glycolytic flux to activation of Ras. Nat Commun. 2017:8:922 